\title{
Le Docteur invraisemblable ou la psychanalyse possible selon Ramón Gómez de la Serna
}

\section{Victor-Arthur Piégay}

\section{(2) OpenEdition \\ 1 Journals}

Édition électronique

URL : http://journals.openedition.org/aes/317

DOI : 10.4000/aes.317

ISSN : 2258-093X

Éditeur

Laboratoire LISAA

Référence électronique

Victor-Arthur Piégay, «Le Docteur invraisemblable ou la psychanalyse possible selon Ramón Gómez de la Serna », Arts et Savoirs [En ligne], 5 | 2015, mis en ligne le 15 mars 2015, consulté le 19 avril 2019. URL : http://journals.openedition.org/aes/317 ; DOI : 10.4000/aes.317

Ce document a été généré automatiquement le 19 avril 2019.

Centre de recherche LISAA (Littératures SAvoirs et Arts) 


\title{
Le Docteur invraisemblable ou la psychanalyse possible selon Ramón Gómez de la Serna
}

\author{
Victor-Arthur Piégay
}

1 L'année 1914 voit la publication, en Espagne, d'un texte intitulé Le Docteur invraisemblable ( El Doctor Inverosímil) dû à la plume de Ramón Gómez de la Serna. Vu l'état d'anonymat quasi absolu dans lequel se trouve Ramón ${ }^{1}$ en France, il n'est peut-être pas inutile de présenter brièvement celui que Valery Larbaud, l'un des deux grands découvreurs de talents ibériques du début du XXe siècle avec Jean Cassou, considérait comme l'égal de Joyce et de Proust. Écrivain proche de multiples avant-gardes, en particulier du Surréalisme, sans n'en avoir jamais été l'un des membres ni avoir pleinement souscrit à leurs doctrines ${ }^{2}$, Ramón s'est essayé, durant une carrière qui s'étend de 1905 pour son premier texte à sa mort en 1963, à tous les genres littéraires, même s'il est surtout reconnu aujourd'hui pour sa poésie. On lui doit en effet l'invention d'un genre nouveau, celui de la greguería, forme poétique brève voisine de l'aphorisme ${ }^{3}$. Mais Ramón s'est aussi essayé au théâtre et aux genres narratifs selon une rigueur générique assez difficile à établir, entre les romans courts (novelas cortas), les romans longs (novelas largas), les « faux romans » (il est l'auteur d'un ouvrage intitulé Seis Falsas Novelas en 19254) et un imposant corpus de nouvelles. 5 . C'est au genre romanesque qu'appartient a priori Le Docteur invraisemblable. La prudence est toutefois de mise car il est bien difficile d'y reconnaître spontanément un roman, bien que le terme employé désormais pour y référer, dans les œuvres complètes de Ramón ${ }^{6}$, soit celui de novela: sans histoire ni intrigue suivie ou linéaire, sans péripéties, sans description du protagoniste ni des personnages secondaires, sans discours intérieur exprimé, le texte se présente comme la compilation d'une centaine de cas cliniques diagnostiqués et traités par le protagoniste, le docteur

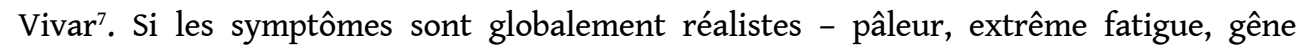
respiratoire, délire, etc. - la méthode utilisée pour les traiter étonne en ce qu'elle ne s'appuie guère sur de quelconques opérations ou médications: le docteur Vivar n'ausculte que rarement les corps, n'opère pas, ne prescrit rien ou presque. C'est plutôt à une véritable investigation qu'il se livre, en observant le patient, son milieu, son 
entourage social, en le questionnant également, pour déterminer la cause de la maladie dont la révélation par la parole au patient constitue le premier pas vers la guérison. Or ce sont bien souvent les objets qui engendrent la maladie: une bibliothèque pleine de poussière suscite ainsi chez l'un l'anhidrose quand une vieille paire de gants entraîne chez un autre une fatigue généralisée. Mais tout invraisemblables qu'ils paraissent, le savoir et la science imaginaires illustrés par Ramón dans son texte apparaissent moins, pour le lecteur du début du XXI siècle, comme des affabulations que des anticipations, la méthode du docteur Vivar s'apparentant, sous bien des aspects, à une forme de psychanalyse servant à soigner des troubles le plus souvent psychosomatiques et ce, avant même la démocratisation du freudisme en Espagne. Il faut préciser que le texte a connu plusieurs états : d'abord novela corta en 1914 (dix chapitres en tout et pour tout), il est devenu en 1921 novela larga après l'adjonction de plusieurs dizaines de cas cliniques nouveaux destinés aussi à nourrir la traduction française du livre, publiée dès 1925. Ces dates sont particulièrement importantes pour replacer le roman de Ramón dans son contexte historique et esthétique. Entre 1914 et 1925, en effet, c'est, d'une part, un savoir réel et nouveau qui se sera démocratisé en Europe - la psychanalyse freudienne - mais également une crise du Naturalisme littéraire qui aura trouvé à se solder se sera soldé par les nouvelles formes romanesques nouvelles développées dans les modernismes européens. Il me semble qu'il est ainsi grand temps de redonner à Ramón la place qui lui revient dans le climat épistémologique de cette décennie bouillonnante. Le Docteur invraisemblable conjugue en effet dans le protagoniste au nom prédestiné une figure de médecin, mais aussi de poète, qui devient l'instrument d'une annonciation et d'une résurrection. Annonçant fictionnellement l'avènement d'un savoir nouveau dans le monde empirique, la psychanalyse, il est aussi celui qui donne une nouvelle vie à la littérature romanesque espagnole.

\section{La médecine imaginaire du docteur Vivar}

2 Au premier abord anti-réaliste, Le Docteur invraisemblable semble se classer sans contredit dans le champ des littératures de l'imaginaire. Si la science sur laquelle repose la pratique du docteur Vivar existe bel et bien dans notre monde - la médecine - c'est surtout la méthode employée par le médecin, mais aussi les causes des maladies qui paraissent invraisemblables. Peut-être convient-il, avant d'examiner deux cas plus en détail, de redonner les définitions de cet adjectif qualificatif qui apparait dans le titre même de l'œuvre. Selon le Trésor de la langue française, est «invraisemblable »: "ce qui n'est pas vraisemblable, ce qui ne semble pas vrai ; ce qui dépasse la commune mesure, ce qui n'est pas dans les normes ; ce qui surprend par son côté extravagant, ridicule $»^{8}$.

3 Les trois définitions peuvent s'appliquer au roman de Ramón et à son personnage, le médecin. Il est en effet peu de dire que la première lecture surprend et que l'impression suscitée par le texte et son personnage est étonnante. Comment considérer Vivar comme un médecin si aucun des sèmes traditionnellement associés à cette profession n'est convoqué ? Vivar ne possède en effet quasi aucun ustensile, ne pratique aucune intervention, «[...] ordonne des choses que l'on ne trouve pas dans les pharmacies $»^{9}$, préférant soigner par la parole. Hors-norme, le «[...] docteur des cas obscurs et désespérés ${ }^{10}$ (14) de Ramón l'est par sa méthode : appelé quand tous les médecins traditionnels ont échoué, il agit comme un véritable détective de la médecine. C'est dire que le genre évoqué par le roman de Ramón est au moins autant celui du récit 
merveilleux que du roman policier ${ }^{11}$. Se déplaçant chez ses clients, scrutant leur environnement et leur passé, se livrant à une véritable enquête, il est un spécialiste de l'étiologie capable de formuler un diagnostic, presque toujours juste, à partir d'une simple observation. Le chapitre "Trichinose de la tête » est à ce titre particulièrement révélateur. Pour soigner le patient, il s'agit de le suivre pour rechercher les causes de sa maladie - son mobile aurait-on envie d'écrire ici - expliquant les symptômes :

«Vous allez continuer à vivre votre vie habituelle, en me permettant de vous accompagner ", lui dis-je en constatant qu'il m'était impossible de trouver l'origine de cette surdité étrange, compliquée du bruit de conversation "d'autres », comme il disait, et suivie de violents maux de tête.

Je le suivis partout. Ses amis ne me parurent pas contagieux, et les endroits où il se rendait pour ses affaires ne semblaient pas infectés.

«Cela commence, me disait-il, vers le milieu de la journée. »

Je cherchais la cause flottante de ce mal ; c'est ce qu'il me fallait trouver, puisque mon client avait déjà été soigné par tous les médecins vraisemblables; il avait pris les remèdes indiqués pour les cas de faiblesse, dérangement d'estomac et neurasthénie qu'on lui avait supposés. ${ }^{12}$ (43)

4 Grâce à cette méthode, qui consiste à suivre le patient dans sa vie quotidienne, à étudier son environnement, le docteur Vivar parvient à découvrir l'origine de la maladie, en l'occurrence les téléphones des cafés qu'utilise assidûment le patient:

Pendant ce temps, je vis l'origine de toute la maladie de mon client.

«Voulez-vous raccrocher et m'écouter?»

Il raccrocha.

«Votre maladie est causée par l'usage que vous faites des téléphones publics, dont l'écouteur est contaminé, sale, plein d'un graillonnement alimenté par d'innombrables oreilles... Abonnez-vous au téléphone, mais ne recommencez pas à avoir recours au téléphone des cafés, dans lesquels se niche la trichine de la tête. $»^{13}$ (44)

5 On voit ici la tonalité juste du roman de Ramón : ni fantastique - il n'y a pas d'hésitation entre naturel et surnaturel - ni étrange - il n'y a pas d'explication rationnelle explicite et la fameuse trichinose, si elle existe bel et bien, s'apparente à un parasite intestinal et non à une infection auriculaire - c'est une forme étonnante de merveilleux qui se met en place et qui passe, si l'on suit Ignacio Soldevila, par l'extravagance, par l'incongruité, par un mécanisme qu'il nomme « invraisemblabilisation » (inverosimilización) qui consiste à créer un écart - même ténu - entre une représentation vraisemblable du réel et ce qu'il appelle «fantaisie visionnaire ${ }^{14}$ ( fantasía visionaria). Dans ce processus, l'humour joue évidemment un rôle prépondérant, de même que des tropes comme les comparaisons, métaphores et personnifications, extrêmement fréquentes chez Ramón - qui créent cette sorte de décrochement, cet à côté du réel, cette autre face, dimension ou perspective, autant de termes visuels appropriés à une littérature parfois qualifiée de cubiste.

6 Le même processus se retrouve dans «L'homme précocement vieilli ». À l'issue de l'investigation menée par le docteur Vivar, le lecteur découvre, en même temps que le personnage malade, la cause des symptômes physiques évoqués dans le premier paragraphe :

La vieillesse précoce de cet ami me déconcertait... Son teint jaune ne provenait pas $\mathrm{du}$ foie ; l'atonie de sa vie ne venait pas non plus du cœur, car il y avait au contraire dans ce cœur une intime jeunesse qui manifestait sa rébellion en palpitations précipitées, soulèvement de tout son être contre un destin collant et parasite.

Je regardais beaucoup mon ami, et, pour le cas où notre amitié normale n'aurait pas suffi à le porter à une confession intime, je lui préparais de ces moments de 
mélancolie où il est nécessaire de tout dire... Mais il y a tant d'erreurs commises à l'ombre que nous ne nous confessons jamais à nous-mêmes, et que nous ne voulons jamais évoquer..$^{15}$ (nous soulignons) (31-32)

7 Ici c'est la montre paternelle léguée au patient, laquelle possède une âme, qui semble nuire à sa santé. La montre permet une nouvelle fois de transfigurer la représentation vraisemblable du réel en ce que l'objet se trouve personnifié par le personnage-narrateur qui va jusqu'à professer une doctrine proche d'une forme d'animisme :

Les montres sont aimantées peu à peu par la vie de celui qui les porte, elles acquièrent les mauvaises habitudes, le tempérament, et la secrète intransigeance de la vie de leur propriétaire... Ta montre a pensé tout près de ton père, elle a connu ses secrets, car, perdue dans la poche de son gilet, elle épiait tous les bruissements de sa vie... Peut-être dans le fin cheveu d'argent qui meut le volant... Peut-être dans le ressort serré et enfermé dans son hermétique écrin en métal... Si tu as démonté une montre, tu dois avoir remarqué la secrète tension du ressort... Et que peut être cette si dilatable tension, sinon de la vie ou de l'âme infuse ? [...]. ${ }^{16}(32)$

Néanmoins, à bien y regarder, l'invraisemblance suscitée par la personnification de la montre et découlant du style poétique que Ramón prête à un Vivar censé être l'auteur de sa propre histoire dans une sorte d'autobiographie professionnelle, masque en fait un trouble qui pourrait tout aussi bien être interprété, à travers le prisme de la psychanalyse freudienne, comme une maladie psychosomatique que Vivar traite selon une méthode qui évoque le freudisme. L'ami souffre en effet de symptômes physiques sans causes physiques, qui ne pourront être soignés que s'il se confesse entièrement au médecin, ce dernier rappelant d'ailleurs au passage que de nombreuses zones d'ombre émaillent notre psyché. Finalement, la maladie du personnage ne serait-elle pas un impossible travail de deuil lié à une figure paternelle obsédante dont la montre constitue le fétiche, rappel constant d'une relation problématique?

9 Je ne suis évidemment pas le premier à remarquer que les maladies soignées et la méthode employée par le médecin évoquent la psychanalyse freudienne tant les preuves abondent dans le texte. Ainsi, dans le chapitre intitulé "La Vieille paire de gants », le recours à la comparaison (dans le texte original que la traduction ci-après ne rend qu'imparfaitement) ne masque pas l'idée d'une importance prépondérante du passé du sujet dans les troubles du présent : « Et rien n'est fatal dans la vie comme la corruption du passé. [...] Le passé corrompt et se digère mal, poisson gâté dont l'arrête est noire. $\aleph^{17}$ (17. La place $\mathrm{du}$ rapport au père réapparaît dans des chapitres comme «L'Homme précocement vieilli » et «Les Papas qui se cachent» tout comme l'importance de la sincérité avec soi dans "L'Homme à barbe": "Il est indispensable d'être toujours sincères dans nos rapports avec nous-mêmes. Sans cela, tant pis pour nous. Nous finirons par nous tuer, sans le savoir, sans que nul s'en aperçoive, mais irréparablement. $~^{18}$ (19)

Dans le même chapitre, on croit aussi lire une forme de simplification en même temps qu'une "poétisation» de la méthode freudienne, notamment par cette mention du danger d'une idée ancrée et cachée dans la psyché, qui finirait par nous nuire : «D'une déviation, d'une idée infectée, de quelque chose qui se durcit, qui se tuméfie, qui se cache au fond de nous, peuvent germer une maladie et une hectisie mortelles. ${ }^{19}$ (19)

On voit également que la recherche des causes du mal passe par la parole. C'est ce qui se produit dans « Le Grand encombrement »: "Voulez-vous vous confesser à moi sans trop réfléchir à ce que vous allez me dire ? $»^{20}(34)$ demande ainsi Vivar, mais aussi dans « La Miss » où il «[...] v[eut] la faire parler pour découvrir et soigner sa nouvelle maladie $»^{21}$ 
(131). Plus simplement, la mention de pathologies réelles semble corroborer l'intuition du lecteur : la phobie dans " La Fausse tâche ", la neurasthénie ou encore l'hystérie.

Difficile après ce relevé, non exhaustif, de penser que Ramón est ignorant en matière de psychanalyse lorsqu'il écrit et publie Le Docteur invraisemblable en 1914 puis en 1921. Le problème qui se pose est qu'il n'est pas certain que Ramón ait eu connaissance de la doctrine du médecin viennois au moment de l'écriture du texte.

\section{La psychanalyse ramonienne : anticipation ou fictionnalisation d'une science ? ${ }^{22}$}

Dans son article intitulé « La psychiatrie espagnole et la psychanalyse des années 1910 à la guerre civile : de la presse médicale au discours social », Anne-Cécile Druet cite les mots de l'historien américain des sciences Thomas Glick : «Parmi les pays où un débat général sur les mérites de Freud eut lieu dans les années 1920 et 1930, l'Espagne a dû être le seul à produire davantage d'analystes fictionnels que réels. $»^{23}$ Ramón serait-il à ce point en avance sur son temps qu'il précéderait les limites relevées par l'historien pour marquer l'existence des analystes fictionnels espagnols ? Bien plus, se livrerait-il à un formidable travail d'anticipation en ayant écrit son roman, dès lors science-fictionnel, sans rien connaître du freudisme? Avant d'énoncer ici les quelques points de vue critiques qui donnent un embryon de réponse à cette question, il faut peut-être commencer par citer Ramón lui-même. En 1941 en effet, lors d'une réédition du Docteur invraisemblable, il fait précéder son texte d'un prologue dans laquelle il revient sur la parenté entre la méthode de son docteur et celle de Freud:

On ne connaissait même pas en Espagne - en dehors de quelques spécialistes de la psychiatrie qui lisaient l'allemand - le nom et la doctrine de Freud, [...] ce n'est donc que bien des années plus tard qu'apparut en Espagne le premier spécialiste de cette toute nouvelle voie de la science. ${ }^{24}$ (nous traduisons)

Puis Ramón d'ajouter qu'il est frappé, à la relecture, de retrouver sa propre version de la psychanalyse, science qui n'était alors protégée par aucun argument d'autorité. À partir de cette déclaration, deux écoles critiques s'affrontent: celle qui considère Le Docteur invraisemblable comme un roman d'anticipation qui ne dit pas son nom et celle qui voit dans le roman la fictionnalisation d'une science balbutiante d'autant plus facile qu'elle est encore peu connue du grand public. Ceux qui, comme Juan Ramón Zaragoza Rubira, croient en la bonne foi de Ramón, et les autres qui, comme Ignacio Soldevila et Rafael Cabañas Alamán, prennent de façon beaucoup plus mesurée la déclaration ramonienne. Pour le premier, le roman de Ramón se présente comme une formidable intuition, « une prédiction de ce que la médecine scientifique découvrira des années plus tard $»^{25}$ (nous traduisons). Cela n'est pas rare dans le domaine de la littérature d'anticipation et Henri Baudin, dans son article intitulé "De l'imaginaire scientifique à la science-fiction", rappelle ainsi que «L'anticipation littéraire peut [...] précéder de loin ce qui n'est pas encore dans l'air du temps. $\aleph^{26}$ Certes, mais que dire quand la science anticipée n'est pas si loin de se trouver dans l'air du temps ? S'il est clair que Ramón n'a pu lire en espagnol les textes de Freud - et pour cause, ils ne seront traduits qu'en 1922 - les nombreux voyages à travers l'Europe de l'écrivain cosmopolite qu'il était, ainsi que la diffusion, ne serait-ce que sous la forme de rumeurs, des découvertes freudiennes, ont sans nul doute participé d'une connaissance, même vague et lacunaire, de la vulgate freudienne. Il est ainsi troublant que, dans sa préface de 1941, Ramón ne mentionne que l'ignorance espagnole 
de la psychanalyse et ne parle pas de la situation des autres pays qu'il a pu visiter et dans lesquels ses œuvres ont rapidement été diffusées, en particulier la France. Évoquant la marginalité supposée des textes freudiens en 1914, Ignacio Soldevila écrit :

Qu'il en fut ainsi en Espagne est probable, mais non à Paris où les essais de Charcot sur l'hypnose pour le traitement de l'hystérie étaient le terreau scientifique dans lequel les théories freudiennes s'enracinèrent et constituèrent l'origine, dès 1885 , des essais de Freud sur l'hystérie et ensuite de son livre sur L'Interprétation des rêves (1900). Toutes proportions gardées, la nouvelle science fut diffusée dans le monde avant 1914, comme le prouve l'existence d'un premier congrès international de psychanalyse en 1908 et les conférences de Freud intitulées "Origine et déroulement de la psychanalyse ", lues aux États-Unis un an plus tard. ${ }^{27}$ (nous traduisons)

Certains détails textuels tendent d'ailleurs à donner raison à Ignacio Soldevila. En effet, comment expliquer, si Ramón ignore tout du freudisme, qu'il connaisse si bien les symptômes de l'hystérie? Dans le chapitre intitulé «La Demoiselle aux costumes écossais ", le personnage-narrateur raconte :

Après avoir reçu le mystérieux avis par lequel s'annonce un nouveau malade, je me présentai dans cette maison croyant avoir affaire à une de ces jeunes hystériques qui simulent les maladies les plus invraisemblables. ${ }^{28}$ (nous soulignons) (47)

Plus loin, dans un chapitre intitulé « Le cadenas à secret » (titre éminemment freudien!), Vivar est amené à soigner un jeune homme dépeint comme fou par ses parents, mais qui souffre plutôt de ce que le docteur nomme "spasme hystérique » engendré par une forme de monomanie, la bonne de la maison déclarant qu' " [i]l ne quitte pas sa chambre, et ne fait que réfléchir comme s'il cherchait à se rappeler quelque chose... $»^{29}(81)$. Visiblement, Ramón connaît certains des symptômes de l'hystérie décrits par Breuer et Freud histrionisme, feinte, mise en scène de soi, volonté de plaire - et sait également que « c'est de réminiscences surtout que souffre l'hystérique $»^{30}$, névrose qui peut aussi se manifester dans des spasmes épileptiques. Mon argument dans ce débat est donc le suivant : Ramón ne connaît pas, en 1914, la totalité de la doctrine freudienne, mais il en connaît suffisamment pour écrire un roman qui, loin d'être une prévision magique, une intuition ou une anticipation, ne constitue que la version fictionnelle d'une science en voie de démocratisation. Il ne faut pas oublier d'ailleurs que le roman est amplement étoffé pour sa réédition en 1921, soit un an seulement avant la publication des œuvres de Freud traduites en espagnol. Nous savons ainsi gré à Rafael Cabañas Alamán, qui dans son ouvrage, en raison même de sa thématique - le fétichisme dans les œuvres de Ramón aborde le rapport de l'auteur à la psychanalyse, de rappeler qu'en

prenant en compte [...] le fait que Gómez de la Serna et Ruiz Castillo [l'éditeur des traductions de Freud] entretenaient de bonnes relations, tant amicales que professionnelles, notre écrivain fut probablement l'un des premiers à posséder les tomes des œuvres complètes avant leur publication en espagnol en 1922 et avant qu'elles n'intègrent les librairies et les bibliothèques de Madrid..$^{31}$ (nous traduisons)

L'on pourra d'ailleurs à bon droit s'étonner, à la lecture du Docteur invraisemblable version novela larga, de repérer les possibles références à la psychanalyse freudienne principalement dans les dix chapitres initiaux, ceux de 1914. L'on pourrait dès lors affirmer que les rapports entre science réelle et science imaginaire sont finalement fortuits, mais le systématisme dans les deux états de publication du roman - le premier saturé de références au freudisme et le second qui tend à les gommer au fur et à mesure que le roman avance - irait plutôt dans le sens de la manipulation concertée d'un Ramón s'ingéniant à brouiller les pistes pour ne pas se voir privé des lauriers paradoxaux 
d'inventeur d'une science existante qu'il ne connaissait pas, mais dont il n'était en réalité peut-être pas si ignorant.

Une donnée resterait à ajouter : le fait, comme le rappelle Anne-Cécile Druet ${ }^{32}$ dans son article, que la découverte progressive de la psychanalyse en Espagne a précisément été moins rapide dans le domaine de la médecine que dans celui de la culture. Elle rappelle ainsi par exemple que le philosophe et essayiste José Ortega y Gasset fit paraître, dès 1911, deux articles consacrés à la psychanalyse : "Psychanalyse : science problématique ${ }^{33}$ et "Nouvelle médecine spirituelle " $^{34}$ (nous traduisons les titres). Là encore, rien n'atteste que Ramón ait lu ces articles, mais il est difficile de croire que cet écrivain avant-gardiste, réunissant périodiquement ses amis intellectuels et cosmopolites au café Pombo de Madrid, ait pu tout ignorer de la psychanalyse et de ce qui apparait, à l'époque, comme l'avant-garde de la science médicale liée au fonctionnement de l'esprit et aux maladies de l'âme.

19 Entre 1911 - date des articles d'Ortega - et 1922 - publication de la première traduction de Freud en espagnol - coexisteraient ainsi trois types de discours sur la psychanalyse en Espagne : un discours scientifique, descriptif et explicatif réservé aux initiés - en résumé, les lecteurs des revues médicales, ces fameux « spécialistes de la psychiatrie qui lisaient l'allemand $»^{35}$ mentionnés par Ramón dans son prologue de 1941 - un discours historique et heuristique, celui d'Ortega qui lève le voile sur ce que la psychanalyse est, et un discours poétique - celui de Ramón - qui donne une idée de ce que la psychanalyse aurait pu être dans un monde possible différent du monde actuel. Mais l'entreprise ramonienne n'est pas que ludique, elle n'est pas qu'une adroite manipulation littéraire destinée à assurer à son auteur le statut de prophète. Il me semble aussi et surtout que Ramón cherche, par la représentation fictionnelle d'une science avant-gardiste, à instaurer un jeu de miroir entre médecine et littérature et à réfléchir sa propre pratique du roman par rapport à ses devanciers.

\section{La science imaginaire au service d'un manifeste pour un nouveau roman}

C'est là peut-être une autre raison expliquant l'utilisation fictionnelle que fait Ramón de cette science balbutiante qu'est la psychanalyse. Il se sert en effet d'un savoir qui pouvait passer, au moment de l'écriture du texte, comme le plus novateur, le plus avant-gardiste. Freud pratique une méthode nouvelle, traite les pathologies psychiques comme personne avant lui. Il en va de même du docteur Vivar qui pratique la médecine comme personne et surtout pas les autres médecins qui «[...] ont de ces idées immuables et fixes dont [il] manque complètement, car [s]a méthode n'est que variété, spontanéité, improvisation $»^{36}$ (23), comme celle de Ramón finalement qui s'apparente à une expérience menée autour du roman davantage qu'à un roman expérimental. La psychanalyse possible de Ramón est aussi un moyen, en filant le lien entre innovation médicale et innovation littéraire, de régler ses comptes avec le Naturalisme. En effet, Ramón s'ancre dans une période touchée, selon Michel Raimond, par une crise du roman naturaliste ${ }^{37}$, et au sein d'un ensemble d'esthétiques contestataires que les Anglo-Saxons appellent modernism et les critiques espagnols, vanguardia : "avant-garde ». C'est dire que le roman de Ramón se pense aussi comme un contrepoint polémique à toute une littérature naturaliste de la fin du XIX ${ }^{e}$ siècle ayant fait de la figure du médecin l'un de ses personnages privilégiés. Cette 
dernière, surtout présente en France, mais dont l'Espagne hérite largement avec un auteur comme Pérez Galdós par exemple, est une littérature scientifique fondée sur une méthode expérimentale - les fameuses hypothèses zoliennes vérifiées par les romans des Rougon-Macquart - mais sur fort peu d'expériences littéraires autour de la forme même $\mathrm{du}$ roman et de ses caractéristiques génériques intrinsèques. Que les choses soient claires : l'objectif de Ramón ici n'est pas uniquement de s'opposer de façon polémique au Naturalisme à partir de la figure du médecin mais surtout, à partir d'une science fictionnalisée, de mettre en question la notion de vraisemblance et le caractère expérimental traditionnellement associés à la littérature naturaliste, le tout, sur les décombres du positivisme du XIX ${ }^{\mathrm{e}}$ siècle.

Dans son article, Juan Ramón Zaragoza Rubira définit ainsi la médecine invraisemblable de Vivar : elle « renvoie à une nouvelle conception de l'homme et de ses relations avec le monde qui l'entoure ${ }^{38}$. D'une façon très ironique, Ramón reprend donc la question du personnage dans son milieu, amplement traitée par Zola, mais oppose à une analyse des structures sociales que l'on pourrait qualifier de macroscopique, une analyse microscopique des détails infimes des relations de l'être humain avec les objets qui forment son environnement et les mouvements imperceptibles de l'âme qui en découlent. Car sous ses dehors extrêmement fantaisistes, le texte de Ramón n'est finalement pas si invraisemblable, pas si irrationnel. Le chapitre liminaire de l'ouvrage intitulé "Présentation", qui met aux prises un narrateur-premier de troisième personne et le docteur Vivar, l'exprime noir sur blanc. Dans un bref dialogue, le narrateur-premier parvient à convaincre le docteur Vivar de devenir l'auteur de sa propre histoire, de livrer une autobiographie professionnelle en insistant, précisément, sur l'aspect rationnel de la méthode du docteur en répondant à Vivar, qui demande pourquoi le narrateur ne raconte pas l'histoire lui-même :

Parce que [...], je défigurerais la raison simple sur laquelle est basé ton procédé. J'abuserais des explications et des descriptions. Je ne résisterais pas au métier, et je mêlerais des éléments romanesques aux claires réalités de ta science..$^{39}(8)$

Ce que craint le narrateur, c'est précisément d'écrire un roman. Naturaliste ? On peut en effet se le demander sachant qu'il est ici question d'explications, de descriptions et de romanesque, mêlés aux données scientifiques. Toutefois, ce que le narrateur premier semble ignorer, c'est que Vivar, devenant un exemple de cette autonomie de la créature fictionnelle qui fait les beaux jours d'une certaine littérature romanesque des premières années du $\mathrm{XX}^{\mathrm{e}}$ siècle en ce qu'il échappe à la volonté de celui qui lui donne la parole, ne livrera pas un vrai roman, mais une suite de récits poétiques, des quasi-poèmes en prose pour le dire autrement. La méthode nouvelle du docteur Vivar pour soigner les malades, ses expériences racontées dans les brefs chapitres qui composent l'œuvre trouvent ainsi à s'exprimer dans un texte qui devient lui-même une expérience, une forme nouvelle, bien loin du Naturalisme romanesque zolien. Tissu de greguerías cousues les unes aux autres, le texte de Ramón tient autant du poème que du roman et évoque, à plus d'un titre, le genre du récit poétique défini par Jean-Yves Tadié. Il semble que ce soit cette nature poétique qui fasse écran à un savoir médical et à des techniques - dans l'un de leurs versants du moins, celui de l'étude des maladies psychiques et psychosomatiques - qui apparaissent ni plus ni moins invraisemblables que les données pseudo-scientifiques alléguées par Zola dans ses textes, pour légitimer certaines de ses scènes parmi les plus spectaculaires, comme la combustion spontanée du vieil oncle Macquart dans Le Docteur Pascal. Dans son ouvrage consacré au récit poétique, Jean-Yves Tadié écrit : 
Le développement du récit poétique au cours de ce siècle et depuis la fin $d u$ Symbolisme [...] correspond au dépérissement progressif du roman classique, dont la survie n'est peut-être que survivance. ${ }^{40}$ un grand éclat de rire poétique, l'instrument d'une annonciation - et participe ainsi, qu'il le veuille ou non, à une entreprise heuristique de dévoilement de la psychanalyse en Espagne - mais aussi de la résurrection d'une littérature romanesque rendue moribonde par un Naturalisme refusant, dans la forme de ses romans, toute expérimentation. C'est ainsi à deux niveaux au moins que se déroule la cure romanesque : pour les patients du docteur Vivar, mais aussi, grâce à la fantaisie de Ramón, pour la littérature.

\section{NOTES}

1. Dans les études qui lui sont consacrées, la convention veut que le patronyme de l'écrivain soit ainsi réduit à son seul prénom. Nous ferons de même dans toute la suite de cet article.

2. La critique a coutume de parler, pour cette période marquée dans la littérature ibérique par la succession des générations $(98,27)$ de " génération unipersonnelle de Ramón Gómez de la Serna » ou encore de « ramonisme » comme s'il était à lui seul, de par l'ampleur et l'impact de son œuvre, un mouvement à part entière.

3. On rappellera l'équation simple proposée par Ramón lui-même pour définir la greguería, poème généralement long d'une ligne ou deux : humour + métaphore = greguería.

4. Le titre de cet ouvrage, malheureusement non traduit, donnerait en français Six faux romans.

5. Sur le rapport entretenu par Ramón avec les formes brèves, on consultera avec profit: Herlinda, Charpentier Saitz, Las Novelle de Ramón Gómez de la Serna, Londres, Tamesis Books, 1990.

6. Fruit d'une mise en ordre salutaire de l'œuvre démesurée de l'auteur, l'édition récente des œuvres complètes de Ramón, dirigée par Ioana Zlotescu, est organisée en huit «espaces littéraires ». Le Docteur invraisemblable ouvre le premier volume de l'espace romanesque : Ramón, Gómez de la Serna, Obras Completas IX, Novelismo I : El Doctor Inverosímil y otras novelas (1914-1923), Barcelone, Galaxia Gutenberg, Círculo de lectores, 1997.

7. La traduction du texte employée dans le présent article, due à Marcelle Auclair, opte pour l'orthographe «Bivar ", en raison, sans doute, de la proximité phonique entre les lettres «b » et « V » en espagnol. Toutefois, nous opterons ici pour l'orthographe originale du nom du protagoniste, "Vivar", mieux à même de traduire la proximité du patronyme avec le verbe espagnol vivir (vivre), perceptible même par un lecteur français non hispanophone en raison de la parenté du verbe avec le français.

8. Paul, Imbs (dir.), Trésor de la langue française, vol. 10, Paris, Éd. du CNRS, 1983, p. 536.

9. Ramón, Gómez de la Serna, Le Docteur invraisemblable, trad. Marcelle, Auclair, Éditions Gérard Lebovici, 1984, p. 41/«[...] recet[a] cosas que no suelen despachar en las boticas» (p. 107). Les références au roman seront désormais placées entre parenthèses après les citations dans le corps 
$\mathrm{du}$ texte et, pour le texte en langue originale, dans les notes. La pagination du texte espagnol renvoie au volume mentionné dans la note $\mathrm{n}^{\circ} 6$.

10. «[...] doctor de los casos desesperados y oscuros» (p. 81).

11. Un passage du chapitre intitulé «Le Savant médecin » est tout aussi révélateur : «Je n'étais pas sans inquiétude en allant chez cet inconnu qui serait certainement surpris ou contrarié de me voir exhumer sa maladie comme qui flaire sans considération la piste d'un crime» ( $p$. 25)/« Me inquietaba la visita a aquel hombre que se había de sorprender o contrariar al verme exhumar su enfermedad como quien olfatea sin consideración la pista de un crimen » (p. 91). Ce roman n'est pas un cas isolé dans la production ramonienne. En effet, le paradigme du roman policier informe de très nombreux textes de Ramón, à l'image de ce fragment de roman écrit par son personnage de romancier fictif Andrés Castilla dans ce qui est sans doute le chef-d'œuvre de la littérature méta-romanesque ramonienne, El Novelista (maladroitement traduit en français par Le Roman du romancier), intitulé « L'Introuvable».

12. «-Usted va a hacer su vida usual y me va a permitir que le acompañe... -le dije al ver que no podía encontrar la causa de aquella extraña sordera, complicada con ruidos de conversaciones «de otros» como él decía, y seguida de violentos dolores de cabeza./ Le acompañé por todos sitios. Sus amigos no parecían ser contagiosos, y en los parejes en que se reunían y en que tramitaban sus negocios no veía yo tampoco el contagio./ -Ahora, ahora me comienza -me decía después de media tarde./ Yo buscaba la causa flotante de aquel mal, porque era la que me correspondía buscar, ya que mi enfermo venía tratado por todos los médicos verosímiles y había tomado las medicinas oportunas por su su mal procedía de la debilidad, del estómago o de la neurastenia» (p. 108).

13. «En ese espacio de tiempo yo vi el origen de toda la enfermedad de mi cliente./ -¿Quiere usted colgar el aparato y oírme a mí? -le dije./ Colgo el aparato./ Todo lo que ha causado su enfermedad es el hablar en los teléfonos públicos de oreja contagiosa, sucia, llena de la grillera alimentada por numerosos oídos... Alquile usted un teléfono, pero no vuelva a utilizar el teléfono de los cafés, en el que anida la trichina de la cabeza» (p. 109).

14. Ignacio Soldevila, «Prólogo» in Ramón, Gómez de la Serna, Obras completas IX, op. cit., p. 44.

15. «La vejez precoz de aquel amigo me tenía desconcertado... Su color cetrino no provenía del hígado; ni la atonía de su vida provenía tampoco del corazón, porque en aquel corazón había, por el contrario, una íntima juventud que revelaba a veces su rebeldía en precipitadas palpitaciones, que eran como escapatorias a un destino pegadizo y advenedizo./ Yo miraba mucho a mi amigo, y por si la normalidad de nuestra amistad no le llevaba a la confesión entrañable, le preparé de esos momentos de melancolía en que es necesario decirle todo... ¡Pero hay tantos errores cometidos en la sombra que jamás se confiesan ni a uno mismo, que jamás se quieren volver a recordar!» (p. 97).

16. "“-Los relojes son inmantados poco a poco por la vida del que los lleva, y adquieren los resabios, el temperamento y la secreta intransigencia de la vida de su dueño... Este reloj tuyo pensó junto a tu padre y se percató de sus secretos, pues perdido en el bolsillo del chaleco espiaba a solas los redaños de su vida... Está lleno de tu padre, aunque no te podría decir dónde radia su parecido y su espíritu... Quizás en la fina hebra de plata que mueve el volante... Quizás en la cuerda encerrada en ese hermético y apretado estuche de metal que guarda la cuerda... Si has desarmado alguna vez algún reloj, habrás visto la tensión secreta que hay en la cuerda... ¿Y qué puede ser esa tensión tan dilatable sino vida o alma infusa?"» (p. 98).

17. «El pasado se corrompe y sienta mal, como un pescado pasado con la espina negra » (p. 84). La traduction transforme en métaphore, et donc en greguería, ce qui, dans le texte original, n'est qu'une simple comparaison. L'originalité poétique de cette formule résiderait plutôt ici dans la polysémie du terme «pasado» («le passé » au sens chronologique et temporel dans la première occurrence, dans son sens adjectival signifiant " gâté, pourri, périmé » dans la seconde) et dans sa parenté phonique avec le nom « pescado » (« poisson »). 
18. «Es necesario que en el trato con nosotros mismos nos portemos con una extrema sinceridad, si no, peor para nosotros. Nos llegaremos hasta a matar, sin saberlo y sin que lo sepa nadie, irreparablemente» (p. 85).

19. «De una torcedura, de una idea enconada, de algo que se quede retestinada, tumefacto, escondido en el fondo de nosotros puede brotar la enfermedad y la hetiquez que mata» (p. 85).

20. «¿Me quiere usted hacer una confesión sin pensar demasiado en lo que haya de decir?» (p 99).

21. «[...] quería [él] que [le] hablase mucho para encontrar y poder tratar la otra enfermedad nueva» (p. 184).

22. L'assimilation de la psychanalyse à une science peut ici étonner. L'objectif du présent article n'est pas d'en débattre dans la mesure où, pour Ramón comme pour José Ortega y Gasset dont il sera question ci-dessous, la nature «scientifique» de la psychanalyse n'est pas à remettre en question.

23. Anne-Cécile, Druet, « La psychiatrie espagnole et la psychanalyse des années 1910 à la guerre civile: de la presse médicale au discours social », El Argonauta Español, Numéro 8, 2011, http:// argonauta.imageson.org/document151.html (consulté le 29 janvier 2015).

24. Ramón, Gómez de la Serna, «Prólogo a la nueva edición» in Obras completas IX, op. cit., p. 71 : «No se conocía aún en España -fuera de algunos especialistas de la psiquiatría que leían el alemán- el nombre y la doctrina de Freud [...] pues hasta muchos años después no aparece en España el primer especialista de ese novísimo camino de la ciencia».

25. Juan Ramón, Zaragoza Rubira, «La medicina inverosímil de Ramón Gómez de la Serna», p. 27, http://institucional.us.es/revistas/rasbl/31/art_2.pdf (consulté le 29 janvier 2015): «una predicción de lo que la medicina científica desarollará años despues».

26. Henri, Baudin, "De l'imaginaire scientifique à la science-fiction », De la science en littérature à la science-fiction, Amiens, Éditions du CTHS, 1996, p. 186.

27. Ignacio, Soldevila, «Una primera etapa de fecundidad narrativa: Ramón se reconcilia con la novela » in Ramón Gómez de la Serna, Obras completas IX, op. cit., p. 48 : « Que así fuera en España es probable. Pero no así en París, donde los ensayos de Charcot con la hipnosis para el tratamiento de histeria fue el humus científico en el que las teorías freudianas arraigaron y dieron origen, ya en 1885, a los ensayos de Freud sobre la histeria y luego a su libro sobre $L a$ interpretación de los sueños (1900). La nueva ciencia estaba difundida en el mundo bastante antes de 1914, como lo prueba la existencia de un primer congreso internacional de psicoanálisis en 1908 y las conferencias de Freud tituladas «Orignen y desarollo del psicoanálisis », leídas en Estados Unidos un año más tarde».

28. «Un día, después de pasarme el aviso misterioso de todo nuevo enfermo, entré en aquella casa, suponiendo a mi enferma una de esas muchachas histéricas que imitan todas las enfermedades inverosímiles» (p. 111).

29. «No sale de su cuarto y no hace más que pensar como queriendo recordar alguna cosa...» (p. 141).

30. Sigmund, Freud, Josef, Breuer, Études sur l'hystérie, trad. Anne, Berman, Paris, PUF, 2007 [1956], p. 5.

31. Rafael, Cabañas Alamán, Fetichismo y perversión en la novela de Ramón Gómez de la Serna, Madrid, Ediciones del laberinto, 2001, p. 139 : «Teniendo en cuenta que [...] Gómez de la Serna y Ruiz Castillo tenían una buena relación amistosa y profesional, nuestro escritor fue probablemente uno de los primeros que hojeó los tomos de las obras completas de Freud antes de la publicación de éstas en español en 1922 y antes de que llegaran a las librerías y bibliotecas de Madrid».

32. Anne-Cécile, Druet, art. cit., http://argonauta.revues.org/142\#ftn9 (consulté le 29 janvier 2015)

33. José, Ortega y Gasset, «Psicoanálisis : ciencia problemática», in Ideas y creencias, Madrid, Revista de Occidente en Alianza Editorial, 2005, p. 89-121. 
34. José, Ortega y Gasset, «Nueva medicina espiritual», in Obras Completas, Madrid, Taurus, t. I, 2004, p. 473-481.

35. Voir infra p. 6.

36. «[...] tienen ideas irremovibles, ideas fijas y tenaces, cuando yo carezco de ellas, por lo variable, lo espontáneo y lo improvisado que es [su] sistema» (p. 89).

37. Michel, Raimond, La Crise du roman : du naturalisme aux années vingt, Paris, José Corti, 1969.

38. Juan Ramón, Zaragoza Rubira, art. cit., p. 29: «un nuevo concepto del hombre y de sus relaciones con el mundo que le rodea».

39. «Porque [...] yo desfiguraría la sencilla razón en que se basan tus procedimientos, excediéndome como escritor en explicaciones y pinturerías. No sabría resistirme a mezclar elementos novelescos a una cosa tan real y tan sencilla como es tu ciencia» (p. 73-74).

40. Jean-Yves, Tadié, Le Récit poétique, Paris, Gallimard, coll. «Tel », 1994, p. 12.

\section{RÉSUMÉS}

En 1914, Ramón Gómez de la Serna publie un roman intitulé Le Docteur invraisemblable dans lequel il donne à lire le rapport entre un savoir imaginaire et un savoir réel en cours de démocratisation. À bien des égards en effet, la méthode employée par le protagoniste éponyme, toute invraisemblable qu'elle paraisse, évoque celle de cette discipline nouvelle qu'est la psychanalyse freudienne. Le roman est ainsi publié durant une période où, si les écrits de Freud n'ont pas encore été traduits, la psychanalyse commence à être connue en Espagne, en particulier grâce aux articles de José Ortega y Gasset. Dans un texte faisant la part belle à la fantaisie et à l'imagination et qui présente de nombreuses caractéristiques du genre du récit poétique, Ramón se livre finalement à la fictionnalisation d'une discipline balbutiante et encore entourée de zones d'ombre.

INDEX

Mots-clés : imaginaire, psychanalyse, Gómez de la Serna (Ramón)

\section{AUTEUR}

\section{VICTOR-ARTHUR PIÉGAY}

Université de Bourgogne 